Supplement of Solid Earth, 11, 173-184, 2020 https://doi.org/10.5194/se-11-173-2020-supplement (C) Author(s) 2020. This work is distributed under the Creative Commons Attribution 4.0 License.

(c) (1)

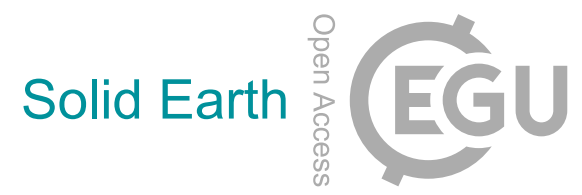

Supplement of

\title{
Observation and explanation of spurious seismic signals emerging in teleseismic noise correlations
}

\section{Lei Li et al.}

Correspondence to: Lei Li (lilei@ies.ac.cn)

The copyright of individual parts of the supplement might differ from the CC BY 4.0 License. 


\section{Computation of correlation function}

Figure S1 describes the computation of the correlation function between two time series. The diagram is self-explained.

\section{Mean-removed series}

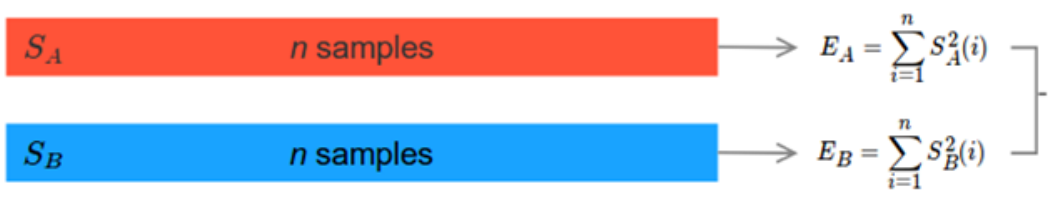

Coherence between $s_{A}$ and $s_{B}$ at lag $\tau$

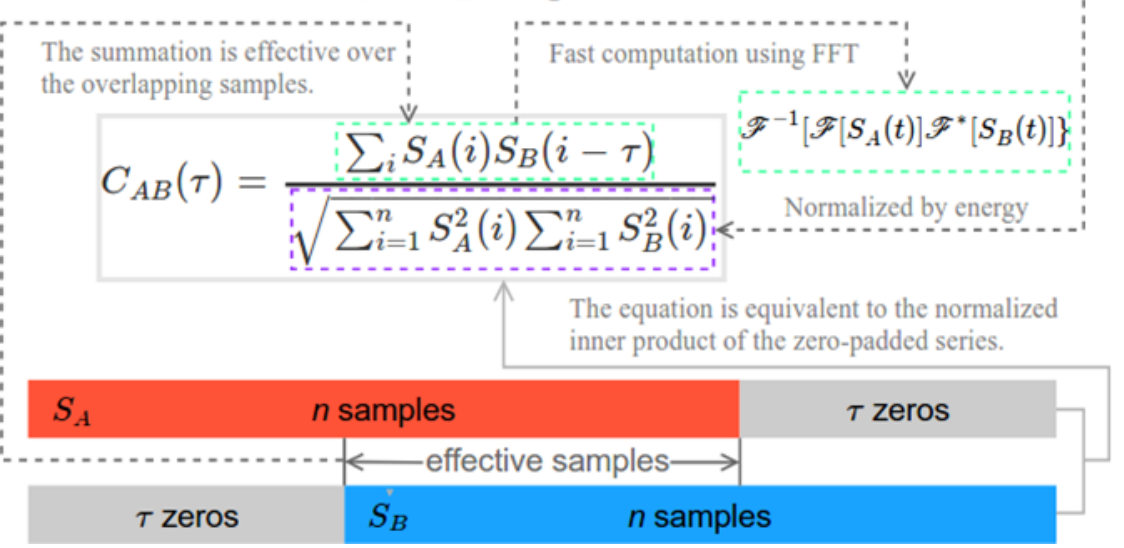

5 Figure S1: Schematic for the computation of the correlation function between two mean-removed time series.

\section{Broadband double-array correlations}

Figure $\mathrm{S} 2$ shows the FNET-LAPNET correlations in the period band of $1 \mathrm{~s}$ to $100 \mathrm{~s}$. The spurious phase is clearly visible in the acausal section. The beamed waveform of the acausal spurious phase and its frequency spectrum are plotted in Fig. S3. It has a peak period of $6.2 \mathrm{~s}$. 

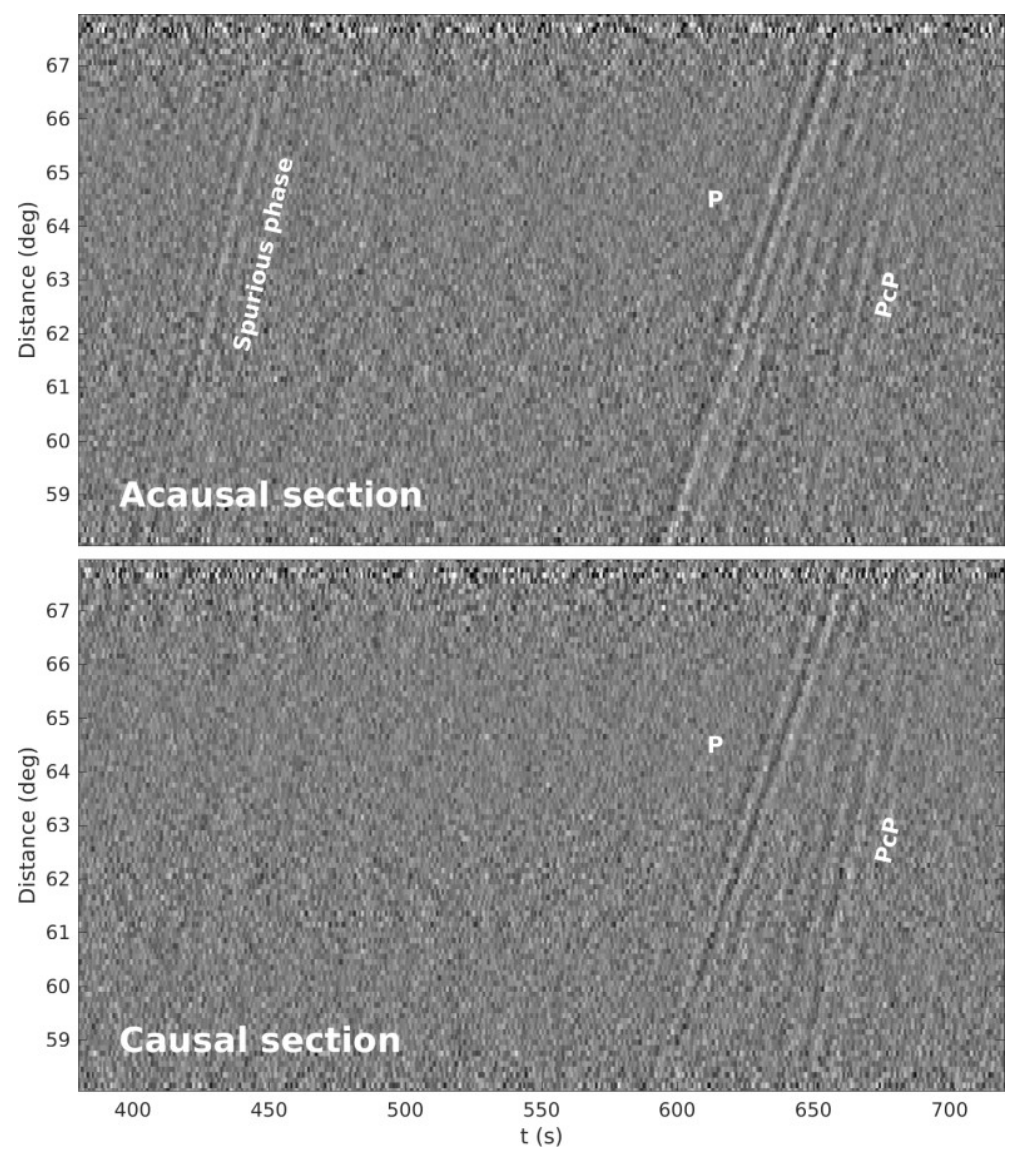

Figure S2: Broadband (1 to $100 \mathrm{~s}$ ) sections of the acausal and causal parts of the vertical-vertical noise correlations stacked in $0.1^{\circ}$ distance bins.
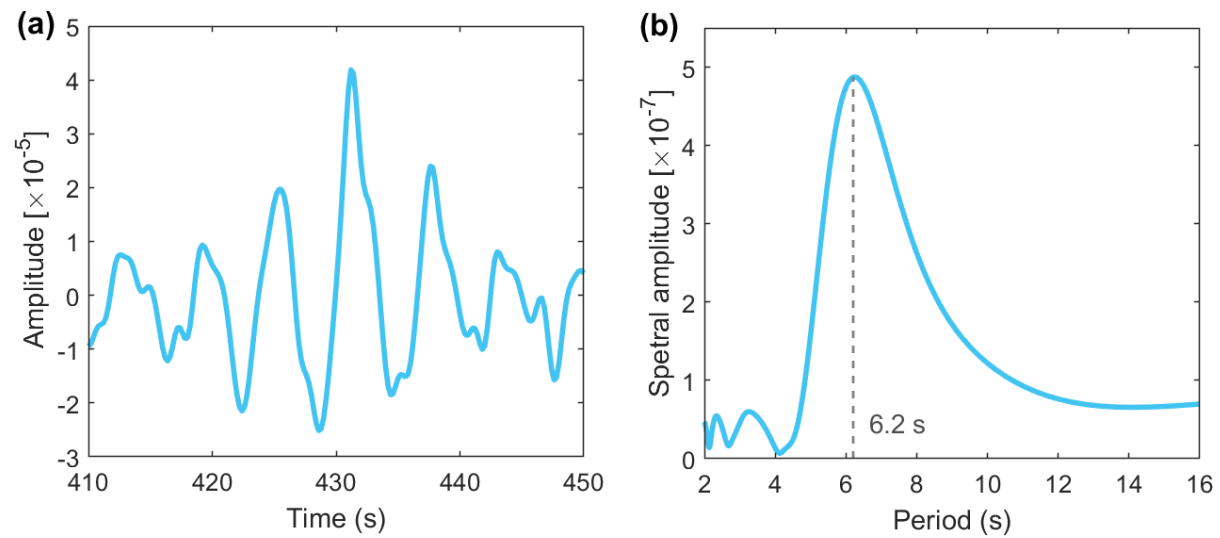

Figure S3: (a) Beamed waveform and (b) amplitude spectrum of the spurious phase in the broadband of 1 to $100 \mathrm{~s}$. 


\section{Stationary-phase examples}

Figure S4 shows the double-array slowness analysis for the noise-derived acausal $\mathrm{P}$ wave and the synthetic experiment for the generation of the inter-receiver P wave from P-PP correlations that can be explained by the traditional stationary-phase arguments, in contrast to the results of slowness analysis for the acausal spurious phase in Fig. 5 and the quasi-stationary-phase

5 explanation in Fig. 9. Slowness analysis in Fig. S4a indicates the correlated waves responsible for the inter-receiver P wave have almost identical slowness. The schematic in Fig. S4b shows that the P wave emanating from the stationary location (label A) to the first receiver and the PP wave to the second receiver have a common slowness and a common P path. The correlation operator cancels the common path and accordingly extracts the phase delay between two receivers. Fig. S4c shows the synthetic source averaging experiment for P-PP correlations. The stationary location corresponds to the extreme point on the dashed

10 time-delay curve. Amplitudes in the P-PP correlations for sources outside the shaded stationary-phase region cancel out by the averaging. The time delay at the maximum of the envelope of the stacked waveform matches exactly with the theoretical travel time of the inter-receiver $P$ wave.

(a) Azimuthal deviation at LAPNET (deg)
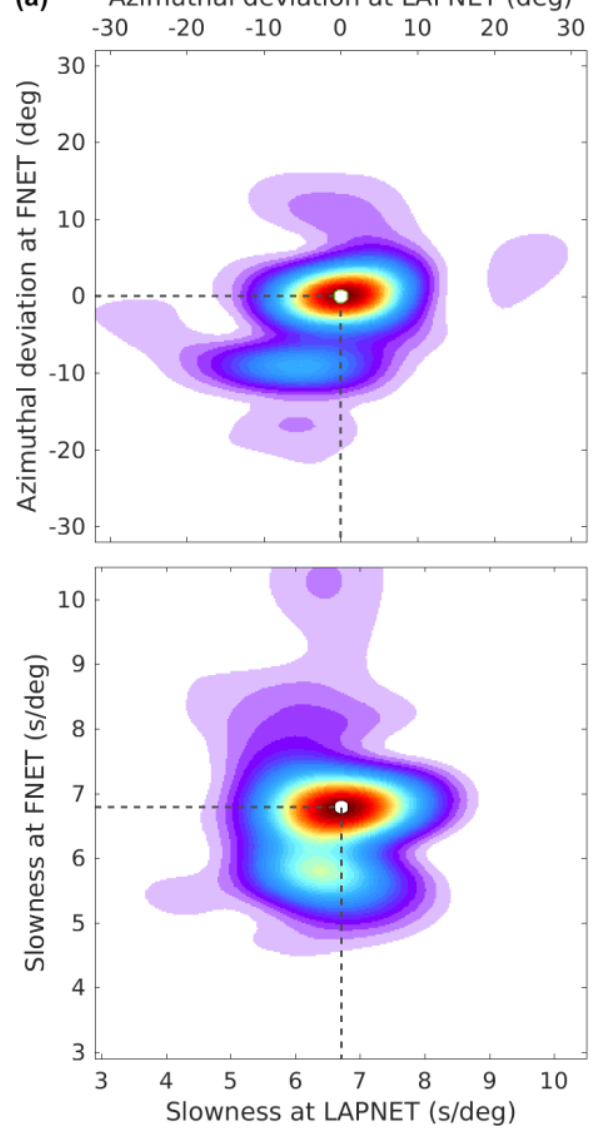

(b)
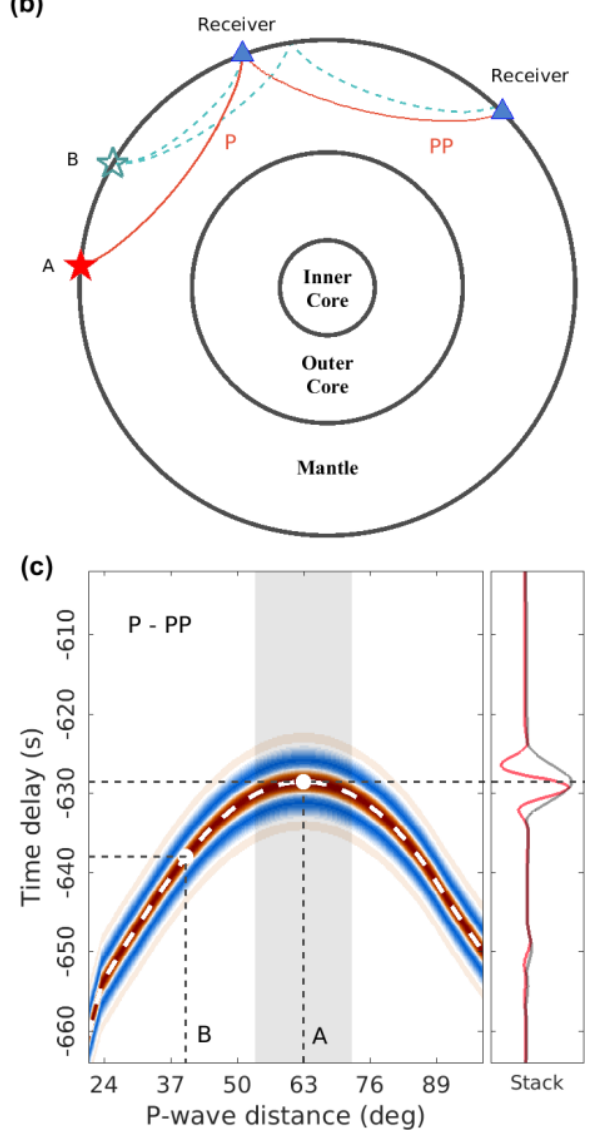
Figure S4: (a) Results of double-array slowness analysis for the acausal $P$ wave. (b) Ray paths of the correlated P and PP waves from distributed sources. Source $A$ is placed at the stationary location. Label B denotes any noise source on the global surface outside the stationary-phase region. For simplicity, the correlations between higher-order multiples like PP-PPP that can also give rise to $\mathbf{P}$ waves are neglected. (c) Reconstruction of the inter-receiver $P$ wave from the P-PP correlations by source averaging, explained by the traditional stationary-phase theory.

In the global section of coda correlations by Phạm et al. (2018), there is also a spurious signal at $\sim 430 \mathrm{~s}$ time delay and $63^{\circ}$ separation distance (see their fig. 2). Phạm et al. (2018) ascribed the signal to the correlation between cS and cPPcP phases for sources distributed on the core-mantle boundary (phase naming in their customized conventions; equivalent to $\mathrm{s}$ and $\mathrm{pPcP}$ phases in IASPEI convention). Here we consider the PcS-PcPPcP correlation, which is an equivalence to their cS-cPPcP correlation but for sources on the global surface. Figure S5 depicts the stationary-phase arguments for the PcS-PcPPcP correlation. However, similar to the discussion about the PcP-PKPab correlation in the main text, the correlated waves are too weak in the ballistic wavefield to be responsible for the spurious phase observed in this study (cf. Fig. 7 in main text). And another strong argument is that, the slownesses of $\mathrm{PcS}$ and $\mathrm{PcPPcP}$ are quite distinct from the slowness estimates in Fig. 2a.
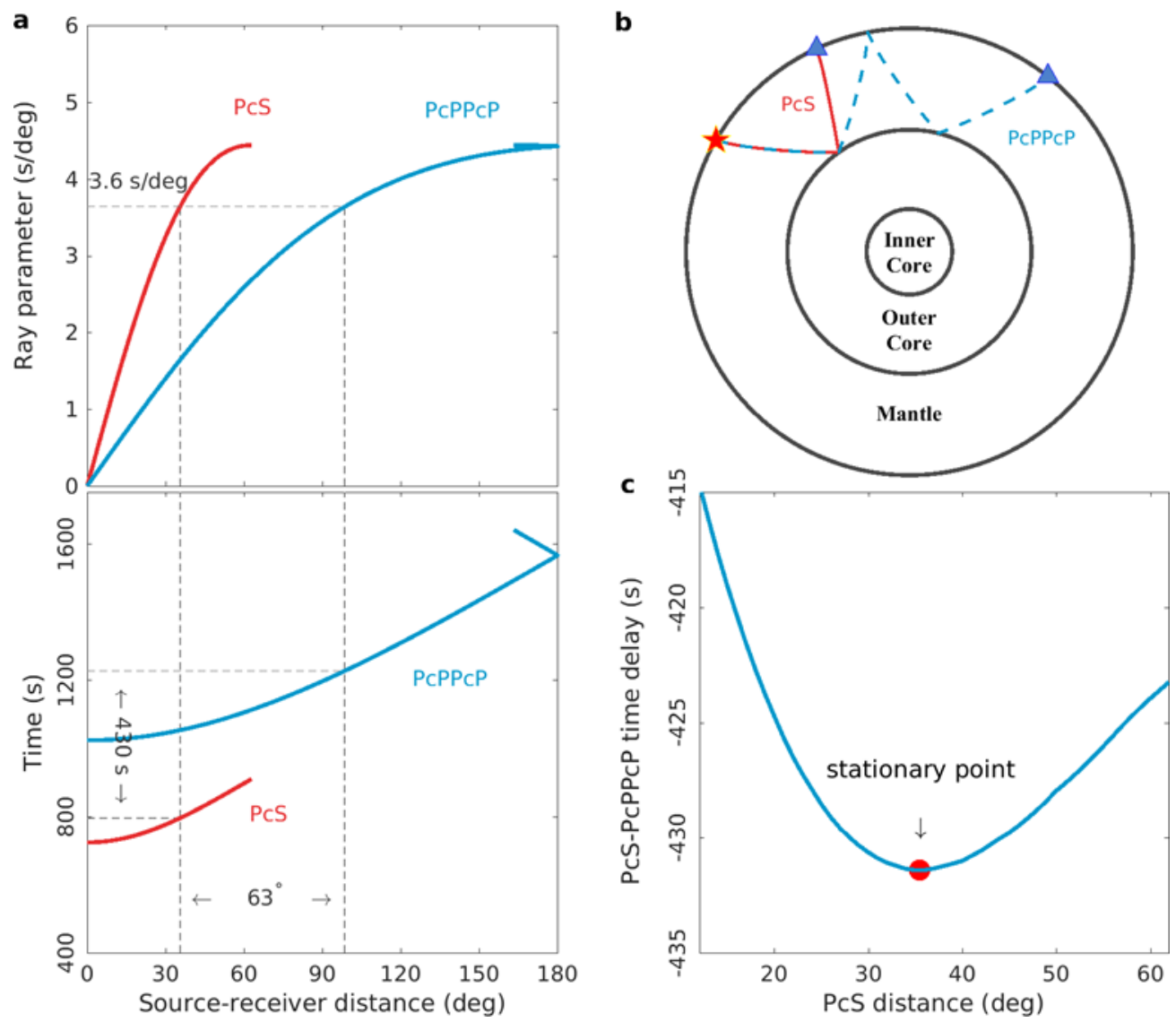

Figure S5: (a) Theoretical curves of ray parameters and travel times of PcS and PcPPcP. (b) Ray paths of PcS and PcPPcP from source at stationary location. (c) PcS-PcPPcP time delays for distributed sources. 


\section{Numerical simulations of P-PKPab correlations}

Figure S6 shows the synthetic P-PKPab correlations for the planar model with sources along the great circle (one-dimensional source distribution) and the spherical model with sources over the global surface (two-dimensional source distribution), using the wave-based method described in section 6 of main text. The synthetic correlations are filtered between $5 \mathrm{~s}$ and $10 \mathrm{~s}$ periods.

5 The spurious phase is visible in a broader distance range in Fig. S6a than in Fig.S6b. The time-distance curves are picked from the maximum on the envelope of each trace. In the common distance range, the two time-distance curves agree well with each other.

(a)

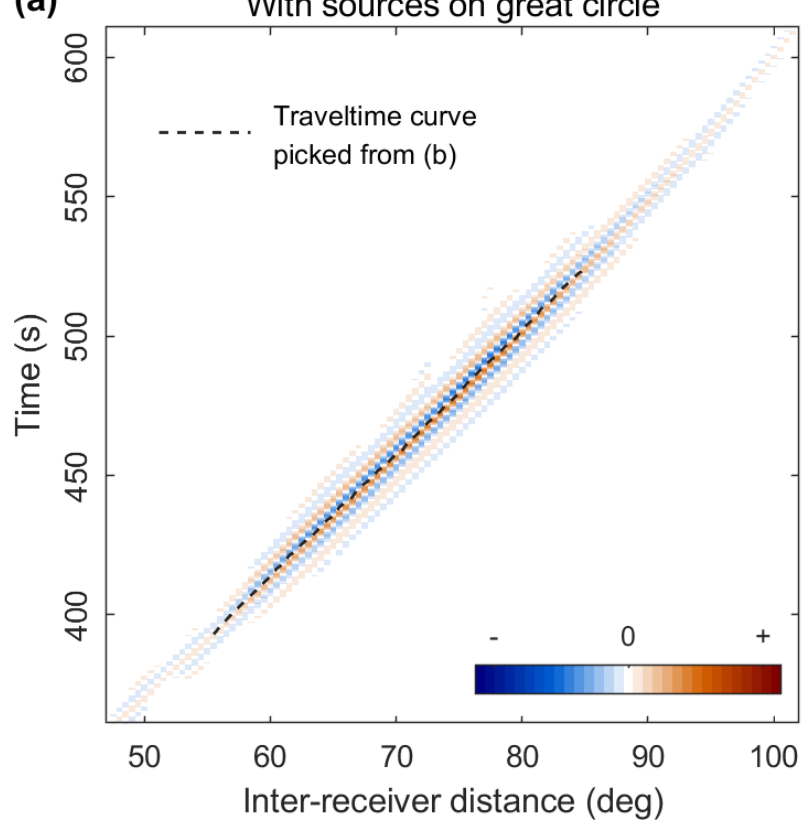

(b)

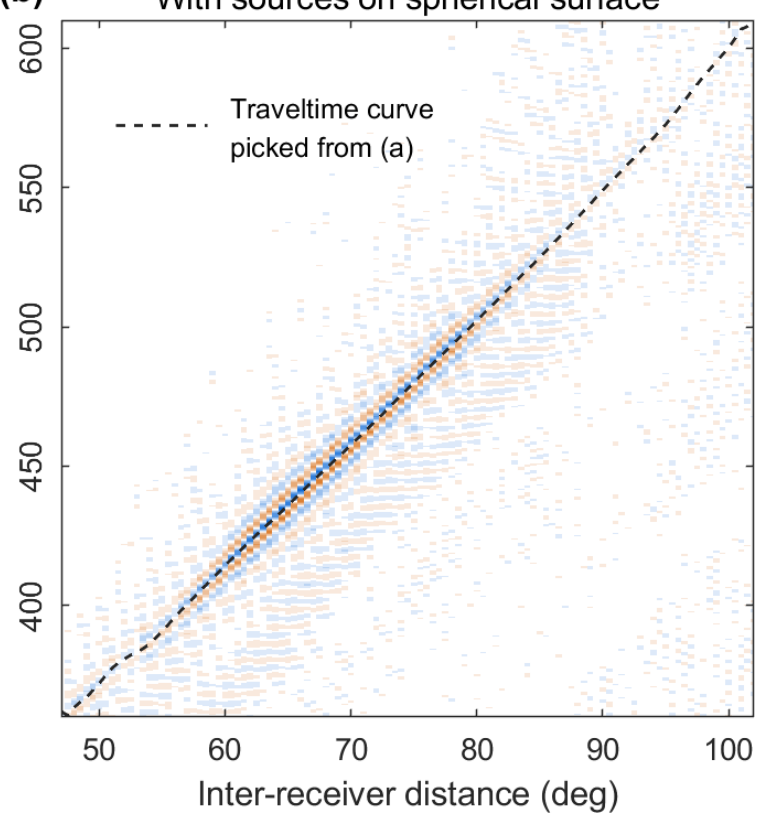

10 Figure S6: Wave-based simulations of P-PKPab correlations with sources (a) on the great circle and (b) on the global surface. 